\title{
Some Common Fixed Point Theorems for Converse Commuting Mappings Via Implicit Relation
}

\author{
XiaO-lan Liu, Sunny Chauhan and Shikha Chaudhari
}

\begin{abstract}
In this paper, we utilize a class of implicit function studied by Imdad et al. [Some common fixed point theorems in Menger PM spaces, Fixed Point Theory Appl. Vol. 2010, Article ID 819269, 14 pages] and prove a common fixed point theorem for converse commuting mappings in Menger space. We give an example which demonstrate the validity of the hypotheses and degree of generality of our main result.
\end{abstract}

\section{INTRODUCTION}

The notion of a probabilistic metric space was first introduced by Menger [14] which is a generalization of metric space. In this theory, the concept of the distance between two points has a probabilistic nature, i.e., it is exhibited by distribution functions. In [31], Sehgal and Bharucha-Reid showed the existence of the fixed point for one-valued local contraction mappings on probabilistic metric spaces. The study of this space was expanded rapidly with the pioneering works of Schweizer and Sklar [30]. The theory of probabilistic space is of fundamental importance in probabilistic functional analysis.

The notion of compatible mappings was introduced by Jungck [9] in 1986. Most of the common fixed point theorems for contraction mappings invariably require a compatibility condition besides continuity of at least one of the mappings. Later on, Jungck and Rhoades [10] studied the notion of weakly compatible mappings and utilized it as a tool to improve commutativity conditions in common fixed point theorems. Many mathematicians proved several fixed point results in Menger spaces (see, for instance $[4,15,16,17,18,19,29])$. In 2002, Lü [12] presented the notion of the converse commuting mappings and established some fixed point theorems for single-valued mappings in metric spaces (also see [13, 26]). Recently, Pathak and Verma [20, 21], Chugh et al. [7], Chauhan and Sapher [6], Chauhan et

2010 Mathematics Subject Classification. Primary: 47H10; Secondary: 54H25.

Key words and phrases. $t$-norm, Menger space, converse commuting mappings, implicit relation, fixed point. 
al. [3] and Wang and Zhu [32] proved some interesting common fixed point theorems for converse commuting mappings in different settings.

In metrical fixed point theory, implicit relations are utilized to cover several contraction conditions in one go rather than proving a separate theorem for each contraction condition. The first ever attempt to coin an implicit relation can be traced back to Popa [22]. Since then many authors modified this class of implicit function and obtained fixed point theorems under weaker conditions (see $[1,2,23,24,25,27]$ ).

In this paper, we prove a unique common fixed point theorem for two pairs of converse commuting mappings in Menger space by using implicit relation due to Imdad et al. [8]. An illustrative and interesting example to highlight the realized improvements is also furnished.

\section{Preliminaries}

Definition 1 ([30]). A $t$-norm is a function $\triangle:[0,1] \times[0,1] \rightarrow[0,1]$ satisfying:

(T1) $\triangle(a, 1)=a, \triangle(0,0)=0$;

(T2) $\triangle(a, b)=\triangle(b, a)$;

(T3) $\triangle(c, d) \geq \triangle(a, b)$ for $c \geq a, d \geq b$;

(T4) $\triangle(\triangle(a, b), c)=\triangle(a, \triangle(b, c))$ for all $a, b, c$ in $[0,1]$.

Examples of $t$-norms are $\triangle(a, b)=\min \{a, b\}, \triangle(a, b)=a b$ and $\triangle(a, b)=$ $\max \{a+b-1,0\}$.

Definition 2 ([30]). A real valued function $F$ on the set of real numbers is called a distribution function if it is non-decreasing, left continuous with $\inf _{u \in \mathbb{R}} F(u)=0$ and $\sup _{u \in \mathbb{R}} F(u)=1$.

We shall denote by $\Im$ the set of all distribution functions defined on $(-\infty, \infty)$ while $H(t)$ will always denote the specific distribution function defined by

$$
H(t)= \begin{cases}0, & \text { if } t \leq 0 \\ 1, & \text { if } t>0\end{cases}
$$

If $X$ is a non-empty set, $\mathcal{F}: X \times X \rightarrow \Im$ is called a probabilistic distance on $X$ and the value of $\mathcal{F}$ at $(x, y) \in X \times X$ is represented by $F_{x, y}$.

Definition $3([14])$. A probabilistic metric space is an ordered pair $(X, \mathcal{F})$, where $X$ is a non-empty set of elements and $\mathcal{F}$ is a probabilistic distance satisfying the following conditions: for all $x, y, z \in X$ and $t, s>0$,

(1) $F_{x, y}(t)=1$ for all $t>0$ if and only if $x=y$;

(2) $F_{x, y}(t)=F_{y, x}(t)$

(3) $F_{x, y}(0)=0$; 
(4) if $F_{x, y}(t)=1$ and $F_{y, z}(s)=1$, then $F_{x, z}(t+s)=1$ for all $x, y, z \in X$ and $t, s \geq 0$.

Every metric space $(X, d)$ can always be realized as a probabilistic metric space by considering $\mathcal{F}: X \times X \rightarrow \Im$ defined by $F_{x, y}(t)=H(t-d(x, y))$ for all $x, y \in X$ and $t \in \mathbb{R}$. So probabilistic metric spaces offer a wider framework than that of metric spaces and are better suited to cover even wider statistical situations, i.e, every metric space can be regarded as a probabilistic metric space of a special kind.

Definition $4([30])$. A Menger space $(X, \mathcal{F}, \triangle)$ is a triplet where $(X, \mathcal{F})$ is a probabilistic metric space and $\triangle$ is a $t$-norm satisfying the following condition:

$$
F_{x, y}(t+s) \geq \triangle\left(F_{x, z}(t), F_{z, y}(s)\right),
$$

for all $x, y, z \in X$ and $t, s \geq 0$.

Definition 5 ([10]). A pair $(A, S)$ of self mappings defined on a non-empty set $X$ is said to be weakly compatible (or coincidentally commuting) if they commute at their coincidence points, i.e., if $A x=S x$ for some $x \in X$, then $A S x=S A x$.

Definition 6 ([12]). A pair $(A, S)$ of self mappings defined on a non-empty set $X$ is called conversely commuting if, for all $x \in X, A S x=S A x$ implies $A x=S x$.

Definition 7 ([12]). Let $A$ and $S$ be self mappings of a non-empty set $X$. A point $x \in X$ is called commuting point of $A$ and $S$ if $A S x=S A x$.

\section{Implicit ReLATION}

Following Imdad et al. [8], let $\Theta$ be the set of all continuous functions $\varphi\left(t_{1}, t_{2}, t_{3}, t_{4}, t_{5}, t_{6}\right):[0,1]^{6} \rightarrow \mathbb{R}$ satisfying the following condition:

$\left(\varphi_{1}\right) \varphi(u, u, 1,1, u, u)<0$, for all $u \in(0,1)$.

Example 1. Define $\varphi\left(t_{1}, t_{2}, t_{3}, t_{4}, t_{5}, t_{6}\right):[0,1]^{6} \rightarrow \mathbb{R}$ as

$$
\varphi\left(t_{1}, t_{2}, t_{3}, t_{4}, t_{5}, t_{6}\right)=t_{1}-\psi\left(\min \left\{t_{2}, t_{3}, t_{4}, t_{5}, t_{6}\right\}\right),
$$

where $\psi:[0,1] \rightarrow[0,1]$ is increasing and continuous function such that $\psi(t)>t$ for all $t \in(0,1)$. Notice that

$\left(\varphi_{1}\right) \varphi(u, u, 1,1, u, u)=u-\psi(u)<0$, for all $u \in(0,1)$.

Example 2. Define $\varphi\left(t_{1}, t_{2}, t_{3}, t_{4}, t_{5}, t_{6}\right):[0,1]^{6} \rightarrow \mathbb{R}$ as

$$
\varphi\left(t_{1}, t_{2}, t_{3}, t_{4}, t_{5}, t_{6}\right)=\int_{0}^{t_{1}} \phi(t) \mathrm{d} t-\psi\left(\int_{0}^{\min \left\{t_{2}, t_{3}, t_{4}, t_{5}, t_{6}\right\}} \phi(t) \mathrm{d} t\right)
$$


where $\psi:[0,1] \rightarrow[0,1]$ is increasing and continuous function such that $\psi(t)>t$ for all $t \in(0,1)$ and $\phi: \mathbb{R}^{+} \rightarrow \mathbb{R}^{+}$is a Lebesgue integrable function which is summable and satisfies

$$
0<\int_{0}^{\epsilon} \phi(s) \mathrm{d} s<1, \quad \text { for all } \quad 0<\epsilon<1, \quad \int_{0}^{1} \phi(s) \mathrm{d} s=1 .
$$

We observe that

$$
\left(\varphi_{1}\right) \varphi(u, u, 1,1, u, u)=\int_{0}^{u} \phi(t) \mathrm{d} t-\psi\left(\int_{0}^{u} \phi(t) \mathrm{d} t\right)<0
$$

for all $u \in(0,1)$.

Example 3. Define $\varphi\left(t_{1}, t_{2}, t_{3}, t_{4}, t_{5}, t_{6}\right):[0,1]^{6} \rightarrow \mathbb{R}$ as

$$
\begin{aligned}
\varphi\left(t_{1}, t_{2}, t_{3}, t_{4}, t_{5}, t_{6}\right)= & \int_{0}^{t_{1}} \phi(t) \mathrm{d} t-\psi\left(\operatorname { m i n } \left\{\int_{0}^{t_{2}} \phi(t) \mathrm{d} t, \int_{0}^{t_{3}} \phi(t) \mathrm{d} t,\right.\right. \\
& \left.\left.\int_{0}^{t_{4}} \phi(t) \mathrm{d} t, \int_{0}^{t_{5}} \phi(t) \mathrm{d} t, \int_{0}^{t_{6}} \phi(t) \mathrm{d} t\right\}\right),
\end{aligned}
$$

where $\psi:[0,1] \rightarrow[0,1]$ is increasing and continuous function such that $\psi(t)>t$ for all $t \in(0,1)$ and $\phi: \mathbb{R}^{+} \rightarrow \mathbb{R}^{+}$is a Lebesgue integrable function which is summable and satisfies

$$
0<\int_{0}^{\epsilon} \phi(s) \mathrm{d} s<1, \quad \text { for all } \quad 0<\epsilon<1, \quad \int_{0}^{1} \phi(s) \mathrm{d} s=1 .
$$

We observe that

$$
\begin{aligned}
& \left(\varphi_{1}\right) \varphi(u, u, 1,1, u, u)=\int_{0}^{u} \phi(t) \mathrm{d} t-\psi\left(\int_{0}^{u} \phi(t) \mathrm{d} t\right)<0, \\
& \quad \text { for all } u \in(0,1) .
\end{aligned}
$$

\section{MAin Results}

Now we prove a unique common fixed point theorem for two pairs of self mappings satisfying a class of implicit function $\Theta$.

Theorem 1. Let $A, B, S$ and $T$ be four self mappings of a Menger space $(X, \mathcal{F}, \triangle)$, where $\triangle$ is a continuous $t$-norm, the pairs $(A, S)$ and $(B, T)$ are conversely commuting respectively satisfying:

(4) $\varphi\left(F_{A x, B y}(t), F_{S x, T y}(t), F_{A x, S x}(t), F_{B y, T y}(t), F_{B y, S x}(t), F_{A x, T y}(t)\right) \geq 0$, for all $x, y \in X, t>0$, and $\varphi \in \Theta$. If $A$ and $S$ have a commuting point, $B$ and $T$ have a commuting point, then $A, B, S$ and $T$ have a unique common fixed point in $X$.

Proof. Suppose that $u$ is the commuting point of $A$ and $S$, then $A S u=S A u$. Also let $v$ be the commuting point of $B$ and $T$, then $B T v=T B v$. Since the mappings $A$ and $S$ are conversely commuting, we have $A u=S u$. Similarly, 
the mappings $B$ and $T$ are conversely commuting, then we have $B v=T v$. Hence $A A u=A S u=S A u=S S u$ and $B B v=B T v=T B v=T T v$.

(i) We assert that $A u=B v$. Suppose that $A u \neq B v$, then using inequality (4) with $x=u, y=v$, we get

$$
\begin{aligned}
& \varphi\left(F_{A u, B v}(t), F_{S u, T v}(t), F_{A u, S u}(t),\right. \\
& \left.\quad F_{B v, T v}(t), F_{B v, S u}(t), F_{A u, T v}(t)\right) \geq 0,
\end{aligned}
$$

or, equivalently,

$$
\varphi\left(F_{A u, B v}(t), F_{A u, B v}(t), 1,1, F_{B v, A u}(t), F_{A u, B v}(t)\right) \geq 0,
$$

which contradicts $\left(\varphi_{1}\right)$. Therefore $A u=B v$. Thus $A u=S u=$ $B v=T v$.

(ii) Now, we show that $A u$ is a fixed point of the mapping $A$. Let, on the contrary, $A u \neq A A u$. On using inequality (4) with $x=A u, y=v$, we have

$$
\begin{gathered}
\varphi\left(F_{A A u, B v}(t), F_{S A u, T v}(t), F_{A A u, S A u}(t),\right. \\
\left.F_{B v, T v}(t), F_{B v, S A u}(t), F_{A A u, T v}(t)\right) \geq 0,
\end{gathered}
$$

and so,

$$
\varphi\left(F_{A A u, A u}(t), F_{A A u, A u}(t), 1,1, F_{A u, A A u}(t), F_{A A u, A u}(t)\right) \geq 0,
$$

which contradicts $\left(\varphi_{1}\right)$. Hence $A A u=A u$. Similarly we assert that $B v=B B v$. If $B v \neq B B v$, then using inequality (4) with $x=u$, $y=B v$, we get

$$
\begin{aligned}
& \varphi\left(F_{A u, B B v}(t), F_{S u, T B v}(t), F_{A u, S u}(t),\right. \\
& \left.\quad F_{B B v, T B v}(t), F_{B B v, S u}(t), F_{A u, T B v}(t)\right) \geq 0,
\end{aligned}
$$

or, equivalently,

$$
\varphi\left(F_{B v, B B v}(t), F_{B v, B B v}(t), 1,1, F_{B B v, B v}(t), F_{B v, B B v}(t)\right) \geq 0,
$$

which contradicts $\left(\varphi_{1}\right)$. Thus $B B v=B v$.

Since $A u=B v$, we have $A u=B v=B B v=B A u$ which shows that $A u$ is a fixed point of the mapping $B$.

On the other hand, $A u=B v=B B v=T B v=T A u$ and $A u=$ $A A u=A S u=S A u$. Hence $A u$ is a common fixed point of $A, B, S$ and $T$.

Uniqueness of the common fixed point is an easy consequence of inequality (4).

Now, we furnish an example which illustrates Theorem 1. 
Example 4. Let $X=[1, \infty)$ with the metric $d$ defined by $d(x, y)=|x-y|$ and for each $t \in[0,1]$, define

$$
F_{x, y}(t)= \begin{cases}\frac{t}{t+|x-y|}, & \text { if } t>0 ; \\ 0, & \text { if } t=0,\end{cases}
$$

for all $x, y \in X$. Define $\triangle(a, b)=\min \{a, b\}$. Clearly $(X, \mathcal{F}, \triangle)$ is a Menger space with $\triangle(a, b)=\min \{a, b\}$. Define $\varphi\left(t_{1}, t_{2}, t_{3}, t_{4}, t_{5}, t_{6}\right):[0,1]^{6} \rightarrow \mathbb{R}$ as

$$
\varphi\left(t_{1}, t_{2}, t_{3}, t_{4}, t_{5}, t_{6}\right)=t_{1}-\psi\left(\min \left\{t_{2}, t_{3}, t_{4}, t_{5}, t_{6}\right\}\right),
$$

where $\psi(s)=\sqrt{s}$ for all $s \in[0,1]$. Let $A, B, S$ and $T$ be self mappings defined by

$$
\begin{aligned}
& A(x)=\left\{\begin{array}{ll}
2 x-1, & \text { if } x<2 ; \\
1, & \text { if } x \geq 2
\end{array} \quad S(x)= \begin{cases}x^{2}, & \text { if } x<2 ; \\
x+3, & \text { if } x \geq 2 .\end{cases} \right. \\
& B(x)=\left\{\begin{array}{ll}
2 x-1, & \text { if } x<2 ; \\
2, & \text { if } x \geq 2 .
\end{array} \quad T(x)= \begin{cases}3 x^{2}-2, & \text { if } x<2 ; \\
x^{2}+1, & \text { if } x \geq 2 .\end{cases} \right.
\end{aligned}
$$

Thus both the pairs $(A, S)$ and $(B, T)$ are each conversely commuting. Thus all the conditions of Theorem 1 are satisfied and 1 is a unique common fixed point of self mappings $A, B, S$ and $T$.

By choosing $A, B, S$ and $T$ suitably, we can derive a multitude of common fixed point theorems for a pair or triod of mappings. As a sample, we obtain the following natural result for a pair of mappings.

Corollary 1. Let $A$ and $S$ be two self mappings of a Menger space $(X, \mathcal{F}, \triangle)$, where $\triangle$ is a continuous t-norm, Suppose that the pair $(A, S)$ is conversely commuting and satisfy:

(5) $\varphi\left(F_{A x, A y}(t), F_{S x, S y}(t), F_{A x, S x}(t), F_{A y, S y}(t), F_{A y, S x}(t), F_{A x, S y}(t)\right) \geq 0$,

for all $x, y \in X, t>0$, and $\varphi \in \Theta$. If $A$ and $S$ have a commuting point, then $A$ and $S$ have a unique common fixed point in $X$.

Corollary 2. The conclusions of Theorem 1 remain true if condition (4) is replaced by one of the following conditions: for all $x, y \in X$ and $t>0$

$$
\int_{0}^{F_{A x, B y}(t)} \phi(t) \mathrm{d} t \geq \psi\left(\int_{0}^{M(x, y)} \phi(t) \mathrm{d} t\right),
$$

where

$$
M(x, y)=\min \left\{F_{S x, T y}(t), F_{A x, S x}(t), F_{B y, T y}(t), F_{B y, S x}(t), F_{A x, T y}(t)\right\},
$$

where $\psi:[0,1] \rightarrow[0,1]$ is a lower semi-continuous function such that $\psi(t)>$ $t$, for all $t \in(0,1)$ along with $\psi(0)=0, \psi(1)=1$ and $\phi: \mathbb{R}^{+} \rightarrow \mathbb{R}^{+}$is a 
summable Lebesgue integrable function such that

$$
\begin{aligned}
& 0<\int_{0}^{\epsilon} \phi(s) \mathrm{d} s<1, \quad \text { for all } 0<\epsilon<1, \text { and } \int_{0}^{1} \phi(s) \mathrm{d} s=1 . \\
& \int_{0}^{F_{A x, B y}(t)} \phi(t) \mathrm{d} t \geq \\
& \psi(\min \{ \int_{0}^{F_{S x, T y}(t)} \phi(t) \mathrm{d} t, \int_{0}^{F_{A x, S x}(t)} \phi(t) \mathrm{d} t, \\
&\left.\left.\int_{0}^{F_{B y, T y}(t)} \phi(t) \mathrm{d} t, \int_{0}^{F_{B y, S x}(t)} \phi(t) \mathrm{d} t, \int_{0}^{F_{A x, T y}(t)} \phi(t) \mathrm{d} t\right\}\right)
\end{aligned}
$$

where

$$
M(x, y)=\min \left\{F_{S x, T y}(t), F_{A x, S x}(t), F_{B y, T y}(t), F_{B y, S x}(t), F_{A x, T y}(t)\right\},
$$

where $\psi:[0,1] \rightarrow[0,1]$ is increasing and continuous function such that $\psi(t)>t$, for all $t \in(0,1)$ and $\phi: \mathbb{R}^{+} \rightarrow \mathbb{R}^{+}$is a summable Lebesgue integrable function such that

$$
0<\int_{0}^{\epsilon} \phi(s) \mathrm{d} s<1, \quad \text { for all } 0<\epsilon<1, \text { and } \int_{0}^{1} \phi(s) \mathrm{d} s=1 .
$$

Proof. The proof of each inequalities (6) and (7) easily follows from Theorem 1 in view of Examples 2 and 3.

\section{ACKNOWLEDGEMEnts}

The authors are thankful to the referee for his useful comments. The work of the first author is partially supported by National Natural Science Foundation of China (Grant No. 11126336), the Scientific Research Fund of Sichuan Provincial Education Department(12ZA098), the Scientific Research Fund of Sichuan University of Science and Engineering (2012KY08).

\section{REFERENCES}

[1] M. Akkouchi and V. Popa, Well-posedness of fixed point problem for mappings satisfying an implicit relation., Demonstratio Math. 43(4) (2010), 923-929.

[2] M. Akkouchi and V. Popa, Well-posedness of fixed point problem for a multifunction satisfying an implicit relation, Math. Moravica 15(2) (2011), 1-9.

[3] S. Chauhan, M.A. Khan and W. Sintunavarat, Fixed points of converse commuting mappings using an implicit relation, Honam Math. J. 35(2) (2013), 109-117.

[4] S. Chauhan and B.D. Pant, Common fixed point theorem for weakly compatible mappings in Menger space, J. Adv. Res. Pure Math. 3(2) (2011), 107-119.

[5] S. Chauhan and B.D. Pant, Fixed points of weakly compatible mappings using common (E.A) like property, LE MATEMATICHE Vol. LXVIII(I) (2013), 99-116. 
[6] S. Chauhan and H. Sahper, Common fixed point theorems for conversely commuting mappings using implicit relations, J. Operat. 2013, Article in press.

[7] R. Chugh, Sumitra and M.A. Khan, Common fixed point theorems for converse commuting maps in fuzzy metric spaces, Internat. Math. Forum 6(37) (2011), 1845-1851.

[8] M. Imdad, M. Tanveer and M. Hasan, Some common fixed point theorems in Menger PM spaces, Fixed Point Theory Appl. Vol. 2010, Article ID 819269, 14 pages. DOI:10.1155/2010/819269

[9] G. Jungck, Compatible mappings and common fixed points, Internat. J. Math. Math. Sci. 9(4) (1986), 771-779.

[10] G. Jungck and B.E. Rhoades, Fixed points for set valued functions without continuity, Indian J. Pure Appl. Math. 29(3) (1998), 227-238.

[11] S. Kumar and B.D. Pant, Common fixed point theorems in probabilistic metric spaces using implicit relation and property (E.A), Bull. Allahabad Math. Soc. 25(2) (2010), $223-235$.

[12] Z. Lü, Common fixed points for converse commuting selfmaps on a metric space, Acta Anal. Funct. Appl. (Chinese) 4(3) (2002), 226-228.

[13] Q.-k. Liu and X.-q. Hu, Some new common fixed point theorems for converse commuting multi-valued mappings in symmetric spaces with applications, Nonlinear Anal. Forum 10(1) (2005), 97-104.

[14] K. Menger, Statistical metrics, Proc. Nat. Acad. Sci. U.S.A. 28 (1942), 535-537.

[15] D. Mihet, A note on a common fixed point theorem in probabilistic metric spaces, Acta Math. Hungar. 125(1-2) (2009), 127-130.

[16] S.N. Mishra, Common fixed points of compatible mappings in PM-spaces, Math. Japonica 36(2) (1991), 283-289.

[17] B.D. Pant and S. Chauhan, A contraction theorem in Menger space, Tamkang J. Math. 42(1) (2011), 59-68.

[18] B.D. Pant and S. Chauhan, Common fixed point theorems for two pairs of weakly compatible mappings in Menger spaces and fuzzy metric spaces, Sci. Stud. Res. Ser. Math. Inform. 21(2) (2011), 81-96.

[19] B.D. Pant, S. Chauhan and Q. Alam, Common fixed point theorem in probabilistic metric space, Kragujevac J. Math. 35(3) (2011), 463-470.

[20] H.K. Pathak and R.K. Verma, Integral type contractive condition for converse commuting mappings, Internat. J. Math. Anal. (Ruse) 3(24) (2009), 1183-1190.

[21] H.K. Pathak and R.K. Verma, An integral type implicit relation for converse commuting maps, Internat. J. Math. Anal. (Ruse) 3(24) (2009), 1191-1198.

[22] V. Popa, A fixed point theorem for mapping in d-complete topological spaces, Math. Moravica 3 (1999), 43-48.

[23] V. Popa, A general coincidence theorem for compatible multivalued mappings satisfying an implicit relation, Demonstratio Math. 33(1) (2000), 159-164.

[24] V. Popa, Some fixed point theorems for weakly compatible mappings, Rad. Mat. 10(2) (2001), 245-252.

[25] V. Popa, On Frigon-Granas type multifunctions satisfying an implicit relation, Demonstratio Math. 40(4) (2007), 907-912. 
[26] V. Popa, A general fixed point theorem for converse commuting multivalued mappings in symmetric spaces, Filomat 21(2) (2007), 267-271.

[27] V. Popa, M. Imdad and J. Ali, Using implicit relations to prove unified fixed point theorems in metric and 2-metric spaces, Bull. Malays. Math. Sci. Soc. (2)33(1) (2010), 105-120.

[28] V. Popa and D. Turkoğlu, Some fixed point theorems for hybrid contractions satisfying an implicit relation, Stud. Cercet. Ştinţ. Ser. Math. Univ. Bacău (1998), No. 8, 75-86.

[29] R. Saadati, D.O'Regan, S.M. Vaezpour and J.K. Kim, Generalized distance and common fixed point theorems in Menger probabilistic metric spaces, Bull. Iranian Math. Soc. 35(2) (2009), 97-117.

[30] B. Schweizer and A. Sklar, Statistical metric spaces, Pacific J. Math. 10 (1960), 313-334.

[31] V.M. Sehgal and A.T. Bharucha-Reid, Fixed points of contraction mappings on probabilistic metric spaces, Math. Systems Theory 6 (1972), 97-102.

[32] P.P. Wang and Chuan Xi Zhu, Common fixed point theorems for converse commuting mappings in an M-PM space, (Chinese) Acta Anal. Funct. Appl. 13(3) (2011), 332336.

Xiao-lan Liu

SCHOOL OF SCIENCE

Sichuan University of Science and Engineering

Zigong, Sichuan 643000

China

E-mail address: stellalwp@163.com

Sunny Chauhan

Near Nehru Training Centre

H. No. 274, Nai Basti B-14

Bijnor-246701, Uttar Pradesh

INDIA

E-mail address: sun.gkv@gmail.com

Shikha Chaudhari

Government Degree College

ChampaWAT-262523, UtTarakHand

INDIA 